\title{
A splitting scheme for three-phase flow models
}

\author{
Hamza Boukili and Jean-Marc Hérard
}

\begin{abstract}
A fractional step method that provides approximate solutions of a threephase flow model is presented herein. The three-fluid model enables to handle smooth or discontinuous unsteady solutions. The numerical method is grounded on the use of the entropy inequality that governs smooth solutions of the set of PDEs. The evolution step relies on an explicit scheme, while implicit schemes are embedded in the relaxation step. The main properties of the scheme are given. Numerical approximations of two basic Riemann problems are eventually presented.
\end{abstract}

Key words: Three-phase flow, entropy, shocks, vapour explosion, finite volumes.

\section{Introduction}

In order to perform numerical simulations of vapour explosion, a phenomenum resulting from the violent interaction between a hot liquid metal and a coolant (usually liquid water and its vapour), flow models with at least three phases are mandatory. Owing to the high velocity and high pressure levels arising in these situations, and also due to the occurence of strong shock waves, models should at least enable highly unsteady simulations, and should be such that unique and well defined jump conditions hold through discontinuities. However, only few contributions arise from the literature on that topic. Among these, one may at least mention $[6,7,8,10,11]$. Actually, we will focus here on the barotropic class defined in [8], and will present a possible fractional step method in order to compute approximate solutions of the

Hamza Boukili ${ }^{1,2}$, Jean-Marc Hérard ${ }^{1,2}$

${ }^{1}$ EDF Lab Chatou, 6, quai Watier, 78400, Chatou, France

${ }^{2}$ I2M, Aix Marseille Université, 39 rue Joliot Curie, 13453, Marseille, France

e-mail: hamza.boukili@edf.fr,jean-marc.herard@edf.fr 
latter model. Some among identified difficulties concern the way to cope with pressure relaxation effects and to preserve positive values of densities and statistical fractions; moreover, schemes should be such that they provide convergent and consistant approximations of shock patterns. Possible extensions to the non barotropic framework and problems arising with mass transfer terms are not adressed here.

\section{Three-phase flow model}

\section{Governing equations}

In the sequel, $\alpha_{k} \in[0,1], \rho_{k}, m_{k}=\alpha_{k} \rho_{k}, U_{k}$, respectively denote the mean statistical fraction, the mean density, the partial mass and the mean velocity of phase $k$ (phase 1 denotes liquid metal). The mean pressure $P_{k}\left(\rho_{k}\right)$ is an increasing function with:

$$
\lim _{x \rightarrow \infty} P_{k}(x)=+\infty \quad ; \quad \lim _{x \rightarrow 0} P_{k}(x)=0
$$

and we note as usual $c_{k}^{2}=P_{k}^{\prime}\left(\rho_{k}\right)$. The set of PDEs that is considered is (see [8]):

$$
\left\{\begin{array}{l}
\frac{\partial \alpha_{k}}{\partial t}+\mathscr{V}_{i}(W) \frac{\partial \alpha_{k}}{\partial x}=\phi_{k}(W) \\
\frac{\partial m_{k}}{\partial t}+\frac{\partial m_{k} U_{k}}{\partial x}=0 \\
\frac{\partial m_{k} U_{k}}{\partial t}+\frac{\partial m_{k} U_{k}^{2}+\alpha_{k} P_{k}}{\partial x}+\Sigma_{l=1, l \neq k}^{3} \Pi_{k l}(W) \frac{\partial \alpha_{l}}{\partial x}=m_{k} S_{k}(W) .
\end{array}\right.
$$

It may be alternatively rewritten in a more condensed form:

$$
\frac{\partial W}{\partial t}+\frac{\partial F(W)}{\partial x}+G(W) \frac{\partial H(W)}{\partial x}=S(W)
$$

where the main variable $W$ and fluxes $F(W), H(W)$ are defined as:

$$
\begin{gathered}
W=\left(\alpha_{2}, \alpha_{3}, m_{1}, m_{2}, m_{3}, m_{1} U_{1}, m_{2} U_{2}, m_{3} U_{3}\right)^{t} \\
F(W)=\left(0,0, m_{1} U_{1}, m_{2} U_{2}, m_{3} U_{3}, m_{1} U_{1}^{2}+\alpha_{1} P_{1}, m_{2} U_{2}^{2}+\alpha_{2} P_{2}, m_{3} U_{3}^{2}+\alpha_{3} P_{3}\right)^{t} \\
H(W)=\left(\alpha_{2}, \alpha_{3}, 0,0,0,0,0,0\right)^{t}
\end{gathered}
$$

$G(W)$ being implicitly defined by (1). The statistical fraction $\alpha_{1}$ complies with: $\alpha_{1}=1-\alpha_{2}-\alpha_{3}$. We restrict herein to the case where: $\mathscr{V}_{i}(W)=U_{1}$, with (see [8]):

$$
\left\{\begin{array}{l}
\Pi_{12}(W)=\Pi_{21}(W)=\Pi_{23}(W)=P_{2} \\
\Pi_{13}(W)=\Pi_{31}(W)=\Pi_{32}(W)=P_{3} .
\end{array}\right.
$$

Closure laws for $\phi_{k}(W), S_{k}(W)$ take the form:

$$
\left\{\begin{array}{l}
\phi_{k}(W)=d(W) \Sigma_{l=1}^{3}\left(\left(P_{k}-P_{l}\right)\right) \\
m_{k} S_{k}(W)=\Sigma_{l=1}^{3}\left(e_{k l}(W)\left(U_{l}-U_{k}\right)\right)
\end{array}\right.
$$


where $d(W)$ and $e_{k l}(W)=e_{l k}(W)$ are positive bounded functions. Meaningful pressure relaxation time scales $d(W)$ arise from [5]. Other relaxation time scales $e_{k l}(W)$ imbedded in momentum transfer terms may be found in the standard literature. We also define: $\psi_{k}^{\prime}\left(\rho_{k}\right)=\frac{P_{k}\left(\rho_{k}\right)}{\rho_{k}^{2}}$, and the entropy of the mixture:

$$
\eta=\Sigma_{k=1}^{3}\left(m_{k} U_{k}^{2} / 2+\psi_{k}\left(\rho_{k}\right)\right),
$$

together with the entropy flux: $f_{\eta}(W)=\Sigma_{k=1}^{3}\left(\frac{U_{k}^{2}}{2}+\psi_{k}\left(\rho_{k}\right)+\frac{P_{k}}{\rho_{k}}\right) m_{k} U_{k}$. Actually this three-phase flow model inherits from similar properties as the Baer Nunziato two-phase flow model [1] (see [2,3] for a slightly broader class).

\section{Main properties}

We recall first the main properties of the latter system (see [8]):

\section{Property 1}

- Structure of the convective subset:

The homogeneous convective subset (left hand side of (2))) is hyperbolic unless $\left|U_{1}-U_{k}\right|=c_{k}$. Its eigenvalues are:

$\lambda_{0,1}(W)=U_{1} \quad ; \quad \lambda_{2,3}(W)=U_{1} \pm c_{1} \quad ; \quad \lambda_{4,5}(W)=U_{2} \pm c_{2} \quad ; \quad \lambda_{6,7}(W)=U_{3} \pm c_{3}$

The 0 - 1-wave is linearly degenerate, while other fields are genuinely non linear.

- Entropy inequality:

Smooth solutions of (2) comply with the entropy inequality:

$$
\frac{\partial \eta(W)}{\partial t}+\frac{\partial f_{\eta}(W)}{\partial x} \leq 0
$$

- Jump conditions:

Within each isolated wave, system (2) admits unique jump conditions.

We may now consider the $0-1$ coupling wave, which is the key point of the homogeneous model. Actually, six independent Riemann invariants arise which are given below. These will be used in order to construct exact solutions of the onedimensional Riemann problem associated with (2) when neglecting source terms.

Proposition 1. Riemann invariants of the $0-1$ coupling wave are:

$$
\begin{gathered}
I_{0,1}^{1}(W)=U_{1} \quad ; \quad I_{0,1}^{2}(W)=m_{2}\left(U_{2}-U_{1}\right) \quad ; \quad I_{0,1}^{3}(W)=m_{3}\left(U_{3}-U_{1}\right) ; \\
I_{0,1}^{4}(W)=\frac{\left(U_{1}-U_{2}\right)^{2}}{2}+\int_{0}^{\rho_{2}}\left(\frac{c_{2}^{2}(x)}{x} d x\right) \quad ; \quad I_{0,1}^{5}(W)=\frac{\left(U_{1}-U_{3}\right)^{2}}{2}+\int_{0}^{\rho_{3}}\left(\frac{c_{3}^{2}(x)}{x} d x\right) ; \\
I_{0,1}^{6}(W)=m_{2}\left(U_{2}-U_{1}\right)^{2}+m_{3}\left(U_{3}-U_{1}\right)^{2}+\Sigma_{k=1}^{3}\left(\alpha_{k} P_{k}\right) .
\end{gathered}
$$


The proof is straightforward though cumbersome.

\section{Numerical scheme}

A fractional step method is introduced in order to compute approximate solutions of (2). The latter method complies with the entropy inequality (5). A Finite Volume scheme is built, considering a classical one-dimensional mesh, where $\Delta x_{i}$ denotes the size of cell $\Omega_{i}$. The first step involves an explicit scheme, whereas the scheme in the second -relaxation- step is implicit.

\section{Time scheme}

- Step 1. A first evolution step computes approximations of solutions of the convective subset; for given $W_{i}^{n}$, the state variable is updated following:

$$
\left\{\begin{array}{c}
\Delta x_{i}\left(W_{i}^{n+1,-}-W_{i}^{n}\right)+\Delta t^{n}\left(\mathscr{F}_{i+1 / 2}\left(W_{i}^{n}, W_{i+1}^{n}\right)-\mathscr{F}_{i-1 / 2}\left(W_{i-1}^{n}, W_{i}^{n}\right)\right) \\
+\Delta t^{n} G\left(W_{i}^{n}\right)\left(\mathscr{H}_{i+1 / 2}\left(W_{i}^{n}, W_{i+1}^{n}\right)-\mathscr{H}_{i-1 / 2}\left(W_{i-1}^{n}, W_{i}^{n}\right)\right)=0 .
\end{array}\right.
$$

- Step 2. The second step takes all source terms into account, for given $W_{i}^{n+1,-}$, and computes $W_{i}^{n+1}$ solution of:

$$
\left(W_{i}^{n+1}-W_{i}^{n+1,-}\right)-\Delta t^{n} \mathscr{S}\left(W_{i}^{n+1,-}, W_{i}^{n+1}\right)=0
$$

where: $\mathscr{S}\left(W_{i}^{n+1,-}, W_{i}^{n+1}\right)=\left(\phi_{i, 2}, \phi_{i, 3}, 0,0,0, S_{i, 1}, S_{i, 2}, S_{i, 3}\right)^{t}$, with:

$$
\phi_{i, k}=\Sigma_{l=1}^{3}\left(d\left(W_{i}^{n+1,-}\right)\left(P_{k}\left(W_{i}^{n+1}\right)-P_{l}\left(W_{i}^{n+1}\right)\right)\right.
$$

and:

$$
S_{i, k}=\Sigma_{l=1}^{3}\left(e_{k l}\left(W_{i}^{n+1,-}\right)\left(U_{l}\left(W_{i}^{n+1}\right)-U_{k}\left(W_{i}^{n+1}\right)\right)\right.
$$

for $k=1,2,3$.

\section{Numerical fluxes in the evolution step}

We restrict herein to simple first-order Rusanov-type fluxes defined as follows:

$$
\mathscr{F}_{i j}\left(W_{i}, W_{j}\right)=\left(F\left(W_{i}\right)+F\left(W_{j}\right)-R_{i j}\left(W_{j}-W_{i}\right)\right) / 2,
$$

together with:

$$
\mathscr{H}_{i j}\left(W_{i}, W_{j}\right)=\left(H\left(W_{i}\right)+H\left(W_{j}\right)\right) / 2
$$

$R_{i j}$ is defined as : $\max _{i, j}\left(r\left(W_{i}\right), r\left(W_{j}\right)\right)$, where $r(W)$ denotes the spectral radius of the whole jacobian matrix $\left(\frac{\partial F(W)}{\partial W}+G(W) \frac{\partial H(W)}{\partial W}\right)$. 


\section{Property 2}

- For given strictly positive values $\left(\alpha_{k}\right)_{i}^{n}$ and $\left(m_{k}\right)_{i}^{n}$, the evolution step computes positive values $\left(\alpha_{k}\right)_{i}^{n+1,-}$ and $\left(m_{k}\right)_{i}^{n+1,-}$ if and only if the time step complies with the classical CFL-like condition:

$$
\Delta t^{n} \max _{j=1 \rightarrow N_{\text {cell }}}\left(R_{j-1 / 2}+R_{j+1 / 2}\right) /\left(2 \Delta x_{j}\right)=C F L<1
$$

- Assume that $\left(\alpha_{k}\right)_{i}^{n+1,-}$ and $\left(m_{k}\right)_{i}^{n+1,-}$ are positive. Then the discrete relaxation Step 2 computes a unique set of positive values $\left(\alpha_{k}\right)_{i}^{n+1}$ and $\left(m_{k}\right)_{i}^{n+1}$, and a unique set $\left(U_{1}, U_{2}, U_{3}\right)_{i}^{n+1}$ without any restriction on the time step.

The proof for the first part involving the evolution Step 1 is classical. Actually, $\left(m_{k}\right)_{i}^{n+1,-}$ is a convex combination of partial masses $\left(m_{k}\right)_{i}^{n}$ and $\left(m_{k}\right)_{i \pm 1}^{n}$, as soon as condition (8) holds. A similar result holds for $\left(\alpha_{k}\right)_{i}^{n+1,-}$. Moreover, when turning to Step 2 , it may be easily checked that the linear system that provides $\left(U_{1}, U_{2}, U_{3}\right)^{n+1}$ admits a unique solution, since the determinant $\delta$ of the local discrete system:

$$
\begin{aligned}
\delta_{i}=m_{1} m_{2} m_{3}+\left(\hat{e}_{13}+\hat{e}_{23}\right) m_{1} m_{2}+\left(\hat{e}_{12}+\hat{e}_{23}\right) m_{1} m_{3}+\left(\hat{e}_{12}+\hat{e}_{13}\right) m_{2} m_{3} \\
+\left(\hat{e}_{12} \hat{e}_{13}+\hat{e}_{12} \hat{e}_{23}+\hat{e}_{13} \hat{e}_{23}\right)\left(m_{1}+m_{2}+m_{3}\right),
\end{aligned}
$$

where $\hat{e}_{k l}$ and $m_{k}$ respectively stand for $\Delta t^{n} e_{k l}\left(W_{i}^{n+1,-}\right)$ and $\left(m_{k}\right)_{i}^{n+1,-}$, is strictly positive. Moreover, the relation $\left(m_{k}\right)_{i}^{n+1}=\left(m_{k}\right)_{i}^{n+1,-}$ guarantees positive values of partial masses. Eventually, the proof of existence and uniqueness of positive values of $\left(\alpha_{k}\right)_{i}^{n+1}$ is more intricate; it requires solving a non linear system with respect to $(x, y)=\left(\left(\alpha_{2}\right)_{i}^{n+1},\left(\alpha_{3}\right)_{i}^{n+1}\right)$ under the constraints: $x>0, y>0,1-x-y>0$. We emphasize that similar schemes have been used for two-phase flow models [9].

\section{Numerical results}

We focus here on simple EOS that read: $P_{k}\left(\rho_{k}\right)=P_{k}^{0}\left(\rho_{k}\right)^{\gamma_{k}}$. Two distinct Riemann problems are investigated, these being representative of what happens in watervapour explosion. The time step complies with the CFL-like condition (8). We have set in all cases: $C F L=1 / 2$. The initial discontinuity separating states $W_{L}$ and $W_{R}$ is located at $x=1 / 2$. We restrict here to uniform meshes, and we consider very large relaxation time scales, setting $d(W)=0$ and $e_{k l}(W)=0$.

Riemann problem 1: The first test case is a classical shock tube problem, where the initial data are such that velocities are null everywhere at the beginning of the computation, whatever the phase is. More precisely, we define $W_{L}$ and $W_{R}$ such that:

$$
\left(\alpha_{2}\right)_{L}=0.4 \quad ; \quad\left(\alpha_{3}\right)_{L}=0.5 \quad ; \quad\left(\alpha_{2}\right)_{R}=0.2 \quad ; \quad\left(\alpha_{2}\right)_{R}=0.3
$$




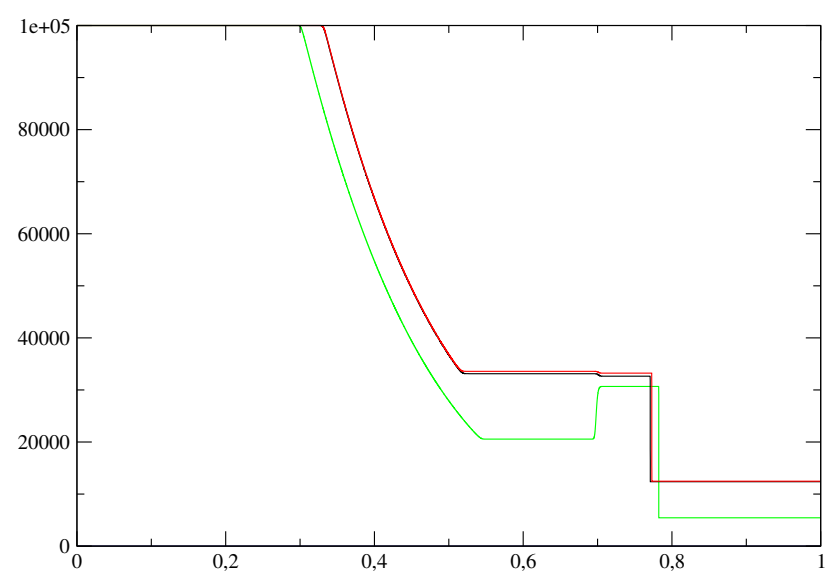

Fig. 1 Riemann problem 1. Pressure profiles on the finest mesh : $P_{1}$ (green), $P_{2}$ (black), $P_{3}$ (red).

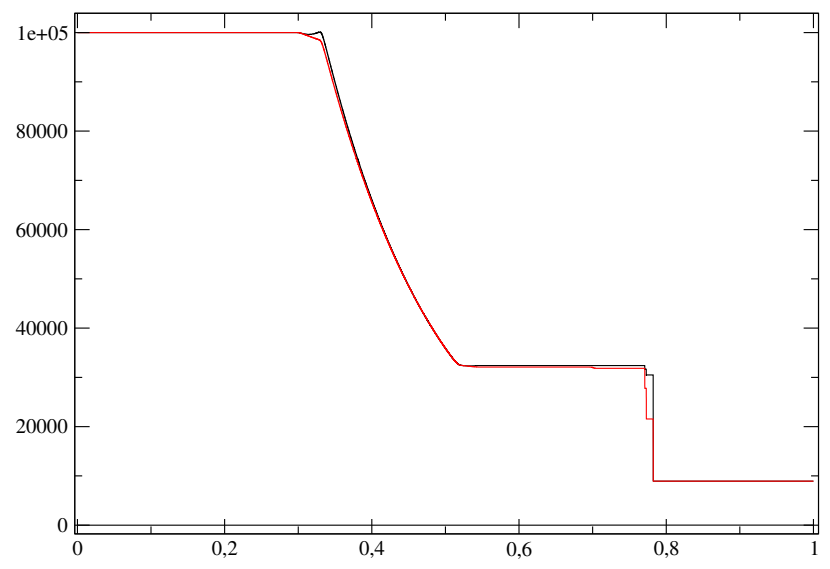

Fig. 2 Riemann problem 1. Pressure profiles on the finest mesh : $\mathscr{P}=I_{0,1}^{6}(W)$ (black), $P_{\text {wall }}$ (red).

$$
\left(U_{k}\right)_{L}=\left(U_{k}\right)_{R}=0 . \quad ; \quad\left(\rho_{k}\right)_{L}=1 . \quad ; \quad\left(\rho_{k}\right)_{R}=1 / 8 .
$$

where EOS are such that: $\gamma_{1}=7 / 5, \gamma_{2}=1.005, \gamma_{3}=1.001$ and $P_{k}^{0}=1.10^{5}$. Phasic pressures are plotted on Figure 1, while $\mathscr{P}=I_{0,1}^{6}(W)$ and the effective pressure of the mixture acting on wall boundaries $P_{\text {wall }}=\Sigma_{k=1 \rightarrow 3} \alpha_{k} P_{k}$ are given on Figure 2 . $\mathscr{P}$ is clearly well preserved through the right-going $0-1$-wave -which is located around $=0.702$ - unlike $P_{\text {wall }}$, which was expected. Velocity profiles have been added on Figure 3. The finest mesh contains 80000 regular cells.

Riemann problem 2: The second test case is a simple Riemann problem where the initial data $W_{L}$ and $W_{R}$ are chosen such that: 


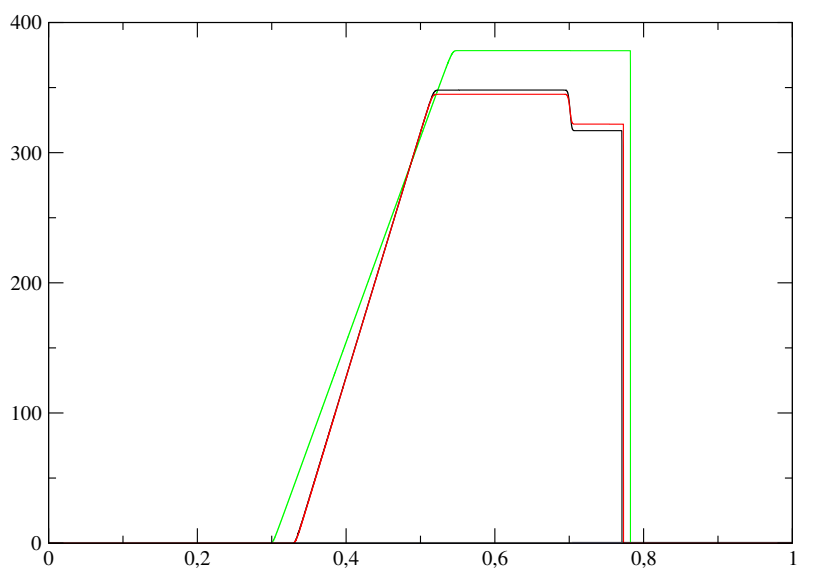

Fig. 3 Riemann problem 1. Velocity profiles on the finest mesh : $U_{1}$ (green), $U_{2}$ (black), $U_{3}$ (red).

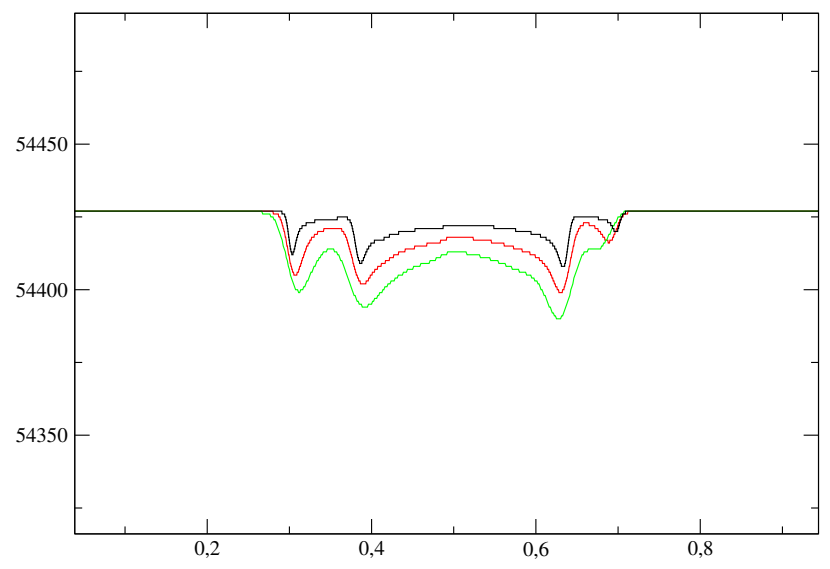

Fig. 4 Riemann problem 2. Pressure profiles for $\mathscr{P}=I_{0,1}^{6}(W)$ on three distinct meshes : 8000 cells (black), 2000 cells (red), 800 cells (green).

$$
I_{0,1}^{m}\left(W_{L}\right)=I_{0,1}^{m}\left(W_{R}\right)
$$

for $m=1 \rightarrow 6$, see property (3). EOS are such that: $\gamma_{1}=3 / 2, \gamma_{2}=2, \gamma_{3}=5 / 2$, and we still set: $P_{k}^{0}=1.10^{5}$. Actually, this is a very tough test case, which is much more discriminating than most of other Riemann problems that involve all waves. A simple though efficient way to measure errors in this particular case consists in computing the $L^{1}$ norm of independent variables $I_{0,1}^{m}(W)$. Obviously, and as expected, the rough Rusanov scheme yields rather high levels of error (close to 0.1 percent on the coarsest mesh, see Figure 4). Nonetheless, and as expected, the error in $L^{1}$ norm varies as $h^{1 / 2}$, since the $0-1$-wave is LD (see Figure 5). 


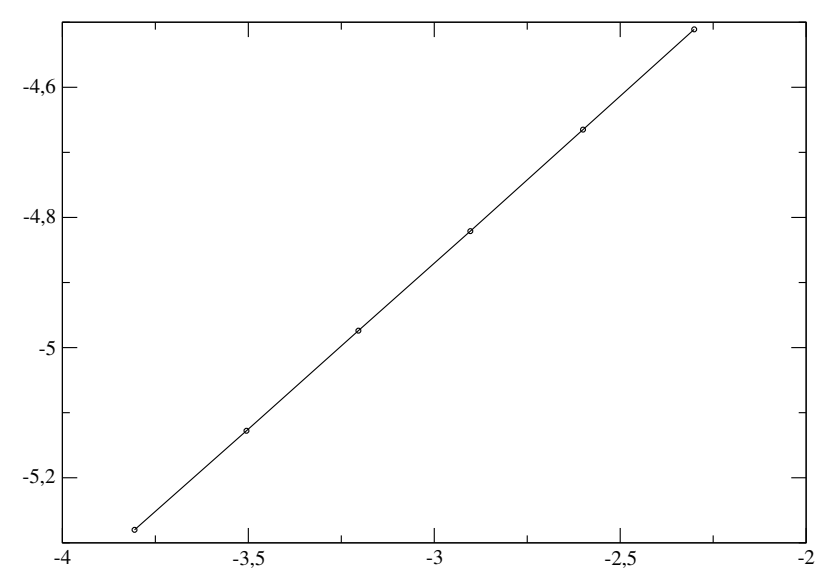

Fig. 5 Riemann problem 2. $L^{1}$ norm of the error for $\mathscr{P}=I_{0,1}^{6}(W)$ vs the mesh size $h$, using log/log scale. Coarsest and finest meshes contain 100 and 6400 regular cells respectively.

Acknowledgements The first author receives financial support by ANRT through an EDF/CIFRE grant number 2016/0611. Computational facilities were provided by EDF.

\section{References}

1. M.R. BAer AND J.W. Nunziato, A two phase mixture theory for the deflagration to detonation transition (DDT) in reactive granular materials, Int. J. Multiphase Flow, vol. 12-6, pp. 861-889, 1986.

2. W. Bo, H. Jin, D. Kim, X. Liu, H. Lee, N. Pestiau, Y. Yu, J. Glimm and J.W. Grove, Comparison and validation of multiphase closure models, Computers and Mathematics with Applications, vol. 56, pp. 1291-1302, 2008.

3. F. Coquel, T. Gallouët, J.M. Hérard and N. Seguin, Closure laws for a two fluid two-pressure model, C. R. Acad. Sci. Paris, vol. I-332, pp. 927-932, 2002.

4. T. FLÄTTEN AND H. LUND, Relaxation two-phase flow models and the subcharacteristic condition. Mathematical Models and Methods in Applied Sciences, vol. 21(12), 2011.

5. S. GAVRILYUK, The structure of pressure relaxation terms: the one-velocity case, EDF report H-I83-2014-0276-EN, 2014

6. S. Giambo and V. La Rosa, A hyperbolic three-phase relativistic flow model, ROMAI Journal, vol. 11, pp. 89-104, 2015.

7. J.M. HÉRARD, A hyperbolic three-phase flow model, Comptes Rendus Mathématique, vol. 342, pp. 779-784, 2006.

8. J.M. HÉrard, A class of compressible multiphase flow models, Comptes Rendus Mathématique, vol. 354, pp. 954-959, 2016.

9. J.M. HÉRARD AND O. HURISSE, A fractional step method to compute a class of compressible gas-liquid flows, Computers and Fluids, vol. 55, pp.57-69, 2012.

10. S. MÜller, M. HANTKE AND P. RICHTER, Closure conditions for non-equilibrium multicomponent models, Continuum Mechanics and Thermodynamics, vol. 28, pp. 1157-1190, 2016.

11. E. Romenski, A. A. Belozerov and I. M. Peshrov, Conservative formulation for compressible multiphase flows, http://arxiv.org/abs/1405.3456, pp. 1-21, 2014. 